\title{
Progress Towards a Predictive Model for Pedestal Height in DIII-D
}

\author{
R.J. Groebner 1), A.W. Leonard 1), P.B. Snyder 1), T.H. Osborne 1), C.F. Maggi 2), \\ M.E. Fenstermacher 3), C.C. Petty 1) and L.W. Owen 4) \\ 1) General Atomics, PO Box 85608, San Diego, California 92186-5608, USA \\ 2) IPP-Garching, Garching, Germany \\ 3) Lawrence Livermore National Laboratory, Livermore, California, USA \\ 4) Oak Ridge National Laboratory, Oak Ridge, Tennessee USA \\ e-mail contact of main author: groebner@fusion.gat.com
}

\begin{abstract}
Recent DIII-D pedestal studies provide improved characterization of pedestal scaling for comparison with models. A new pedestal model accurately predicts the maximum achieved pedestal width and height in TYPE-I ELMing discharges over a large range of DIII-D operational space, including ITER demonstration discharges. The model is a combination of the peeling-ballooning theory for the MHD stability limits on the pedestal with a simple empirical pedestal width scaling, which is proportional to the square root of the pedestal poloidal beta. Width scalings based on the ion toroidal or poloidal gyroradius are much poorer descriptions of DIII-D data. A mass scaling experiment in $\mathrm{H}$ and $\mathrm{D}$ provides additional support for a poloidal beta scaling and is not consistent with an ion poloidal gyroradius scaling. Studies of pedestal evolution during the inter-ELM cycle provide evidence that the pedestal width and height increase during pedestal buildup. These studies show a correlation of pedestal width with both the square root of the pedestal poloidal beta and the square root of the pedestal ion temperature during the pedestal buildup.
\end{abstract}

\section{Introduction}

Developing a predictive capability for the height of the H-mode pedestal remains an important issue for predicting the performance of ITER [1]. The DIII-D pedestal program has placed a significant emphasis on making improved characterizations of the pedestal width and height and comparing the results to pedestal models. This research has led to the development of an empirical scaling of the width with the square root of the pedestal poloidal beta, $\left(\beta_{\theta}^{\text {ped }}\right)^{1 / 2}[2]$, which is a good description of scaling results from DIII-D experiments. The width scaling has been combined with a model of the peeling-ballooning MHD stability theory to produce a predictive model for both pedestal height and width [2]. Tests of this model show that it predicts these parameters with good accuracy in DIII-D in an experiment where the pedestal height was varied by more than a factor of ten. This model also provides good predictions for the pedestal height of discharges made with the proposed ITER shape, safety factor and beta. Thus, this model forms a very good basis for further predictions in DIII-D and potentially for ITER

Scalings of pedestal width against the ion toroidal or poloidal gyroradius show much more scatter than scalings against $\left(\beta_{\theta}^{\text {ped }}\right)^{1 / 2}$. In addition, a mass scaling experiment showed that the widths were nearly identical in $\mathrm{H}$ and $\mathrm{D}$ discharges with very similar pedestals. This is consistent with a $\left(\beta_{\theta}^{\text {ped }}\right)^{1 / 2}$ scaling and inconsistent with an ion gyroradius scaling. Nevertheless, recent studies provide evidence of the pedestal width increasing with time during the ELM cycle and of the increase being correlated with an increase in both $\left(\beta_{\theta}^{\text {ped }}\right)^{1 / 2}$ and the square root of the pedestal ion temperature. The relationship between pedestal width and $\left(\beta_{\theta}^{\text {ped }}\right)^{1 / 2}$ during the ELM cycle is similar to the relationship observed just prior to the ELM crash. 


\section{Test for a Model for Pedestal Height}

Recently, the DIII-D pedestal program tested a new model [2] for the H-mode pedestal height. A fundamental ingredient of this model is the peeling-ballooning theory for the onset of Type-I ELMs in standard ELMing H-mode discharges. Previous studies have shown that the peeling-ballooning theory can be used to successfully predict the experimentally observed pedestal pressure achieved at the onset of an ELM instability, when the measured pedestal width is used as an input to a model calculation [3]. The new theoretical model, called EPED1 [2], uses an empirically derived scaling for the pedestal width $\Delta$ in place of the measured width. The new empirical scaling is $\Delta=0.076\left(\beta_{\theta}^{\text {ped }}\right)^{1 / 2}$ where $\Delta$ is measured in normalized poloidal flux, $\beta_{\theta}^{\text {ped }}=2 \mu_{0} p^{\text {ped }} / B_{\theta}^{2}$ is the total pedestal poloidal beta, $p^{\text {ped }}$ is the total pedestal pressure and $B_{\theta}$ is the flux-surface average poloidal magnetic field at the separatrix. In this scaling, $\Delta$ is defined as the average of the widths of the electron density and temperature profiles, as determined with a fit to a modified tanh function [4]. All width parameters used here are the full pedestal widths and are obtained from twice the width parameter used in the tanh function [4]. The total pedestal pressure $p^{\text {ped }}$ is taken as $2 n_{\mathrm{e}}^{\text {ped }} T_{\mathrm{e}}^{\mathrm{ped}}$, where $n_{\mathrm{e}}^{\text {ped }}$ and $T_{\mathrm{e}}^{\text {ped }}$ are the pedestal heights for the electron density and temperature profiles. Thus, this scaling is determined with the assumption that the pedestal ion temperature $T_{\mathrm{i}}^{\text {ped }} \approx T_{\mathrm{e}}^{\text {ped }}$ and that pedestal main ion density $\approx n_{\mathrm{e}}^{\text {ped }}$. This scaling is very similar to a pedestal width scaling obtained in 1999 [5], $\Delta_{\mathrm{pe}} \propto\left(\beta_{\theta}^{\mathrm{ped}}\right)^{0.4}$, where $\Delta_{\mathrm{pe}}$ is the electron pressure width. The new scaling is in fact based on an analysis of much of the same data set used in the 1999 work as well as a significant amount of post-1999 data. The database covers a wide range of plasma currents, toroidal fields and input heating powers for discharges in the original ITER shape. Both the 1999 and the new scaling were developed from pedestal measurements just before the onset of a Type I ELM.

\subsection{Test of Pedestal Width Scaling}

Pedestal experiments performed in the last two years on DIII-D have typically used a statistical approach to obtain pedestal parameters. This approach often provides clear trends in pedestal parameters from a small number of discharges. This analysis method is based on fits of the modified tanh fit function to composite profiles, which are obtained from measurements at several times during a long steady state phase of a discharge [6]. ELM-detection techniques, based on measurements of $D_{\alpha}$ light, are used to select multiple data sets, all from the same phase of an ELM cycle, the period between the end of one ELM and the start of the next ELM. Typical analysis of DIII-D discharges uses $80 \%-99 \%$ of the ELM cycle.

Pedestal parameters in DIII-D, obtained with these statistical techniques, are well described by the new width scaling. This scaling has been examined in a variety of discharge conditions in which scans of neutral-beam heating power and changes in plasma shape were used to vary the beta of the pedestal [7]. Power scans were used to increase the global and pedestal beta in hybrid and advanced tokamak discharges. In these scans, increases of power usually caused an increase in the pedestal beta $\beta^{\text {ped }}$. The pedestal pressure width was observed to increase with $\beta^{\text {ped }}$ in these scans and this variation is well described by the width scaling $\Delta \sim\left(\beta_{\theta}^{\text {ped }}\right)^{1 / 2}$. Shape scans were also used to vary $\beta^{\text {ped }}$ at nearly constant global beta. 
These scans included a scan of squareness, in which $\beta^{\text {ped }}$ decreased with increased squareness, and a scan of triangularity, in which $\beta^{\text {ped }}$ increased with increased triangularity. In both scans, the pedestal width scaling was well described by $\Delta \sim\left(\beta_{\theta}^{\text {ped }}\right)^{1 / 2}$. Thus, in several scans where pedestal beta was varied, the pressure width changed as expected from the new width scaling, as will be shown in the next section. An increase of the electron temperature width $\Delta_{T \mathrm{e}}$ with $\left(\beta_{\theta}^{\text {ped }}\right)^{1 / 2}$ has also been observed [8]. However, the data were also consistent with a linear dependence of $\Delta_{T \mathrm{e}}$ on $\left(\beta_{\theta}^{\text {ped }}\right)^{1 / 2}$.

\subsection{Integrated Test of Model}

The inputs to the EPED1 model include the magnetic equilibrium parameters $B_{\mathrm{T}}, I_{\mathrm{p}}, R, a$, $\mathrm{\kappa}$, $\delta$, which are, respectively, toroidal field, plasma current, major radius, minor radius, elongation and average triangularity. The plasma inputs to the model are $n_{\mathrm{e}}^{\text {ped }}$ and the total plasma beta-poloidal $\beta_{\theta}$. In addition, there are fixed assumptions about the shapes of temperature and density profiles. The model is used to predict the pedestal pressure width and height, achieved just before the onset of an ELM in a standard Type-I ELMing discharge.

The model implies that significant variations of pedestal height could be obtained in DIII-D through variations of $B_{\mathrm{T}}, I_{\mathrm{p}}$, and $\delta$. An experiment was designed to vary these parameters significantly and to obtain the statistical pedestal datasets, discussed in Sec. 2.1. The experiment was designed to maintain the normalized toroidal global beta $\beta_{\mathrm{N}}$ at about 2.0 and $n_{\mathrm{e}}^{\text {ped }}$ at $0.4-0.6$ of the Greenwald density. The scans included variations of $I_{\mathrm{p}}$ from 0.5-1.5 MA at $B_{\mathrm{T}}=2.1 \mathrm{~T}$ at the two triangularities $\delta=0.2$ and 0.55 , scans of $I_{\mathrm{p}}$ at fixed $q$ with $I_{\mathrm{p}}=0.5-1.5 \mathrm{MA}, B_{\mathrm{T}}=0.7-2.1 \mathrm{~T}$ at $\delta=0.2$ and $\delta=0.55$ and a scan of $\delta=0.2-0.55$ with $I_{\mathrm{p}}=$ $1.16 \mathrm{MA}, B_{\mathrm{T}}=2.1 \mathrm{~T}$.

The EPED1 model was used to predict the pedestal heights for the discharges in the plan prior to the execution of the experiment. When the experiment was performed, the pedestal pressure was varied by more than an order of magnitude due to changes in $B_{\mathrm{T}}, I_{\mathrm{p}}$, and $\delta$. The model predictions agreed with experiment to within $\sim 10 \%-15 \%$. However, the control parameters obtained in the actual experiment were slightly different than those used in the pre-experiment predictions. The EPED1 model was rerun with the actual values of the control parameters $\delta$,

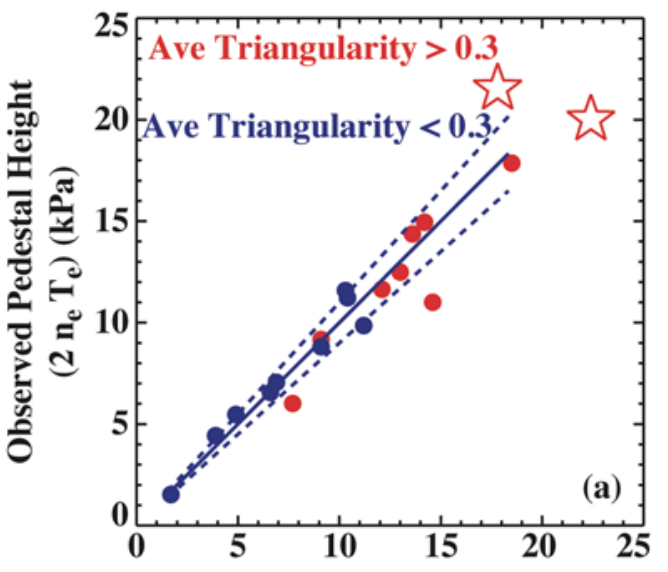

Predicted Pedestal Height (EPED1, kPa)

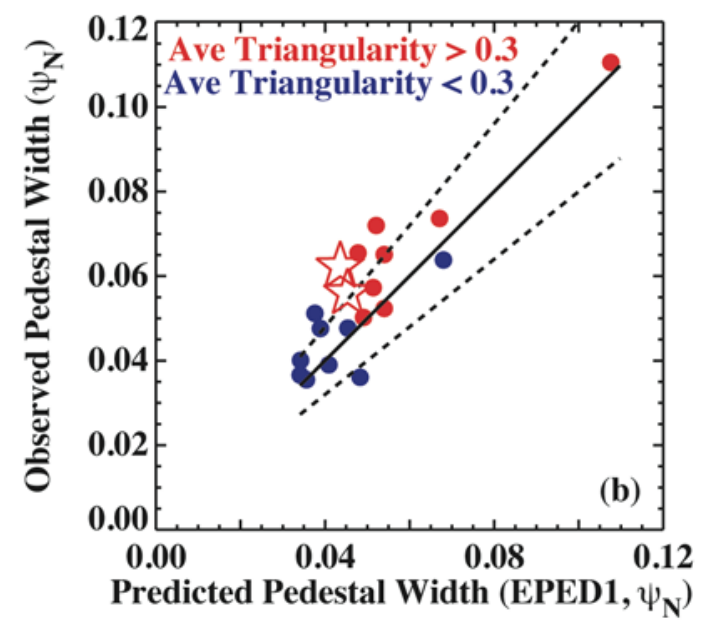

FIG. 1. Measured pedestal height (a) and measured width (b) vs predictions of EPED1 model. Open stars from ITER demo discharges. Solid lines are unity lines. Dashed lines show deviations of $\pm 10 \%$ and $\pm 20 \%$ from unity in (a) and (b), respectively. 
$n_{\mathrm{e}}^{\text {ped }}$ and global $\beta_{\theta}$ achieved in the experiment and even better agreement was obtained with the experiment, as shown in Fig. 1. Figure 1(a) shows that the predicted total pedestal pressure height from the model agrees well with the measured pedestal pressure over more than a factor of ten. (The circles in Fig. 1 are from the experiment to test the model. The open stars are from ITER demonstration discharges and will be discussed later.) The average ratio of predicted to observed height is $1.03 \pm 0.13$, with the observed height evaluated from $2 n_{\mathrm{e}}^{\text {ped }} T_{\mathrm{e}}^{\text {ped }}$. Figure 1 (a) also shows that the highest pressures were achieved at the higher triangularities. The predicted pedestal widths from the EPED1 model calculations also compare well to the experimental widths, computed from $0.5 *\left(\Delta_{\mathrm{ne}}+\Delta_{\mathrm{Te}}\right)$, where $\Delta_{\mathrm{ne}}$ and $\Delta_{\mathrm{Te}}$ are the measured pedestal widths for electron density and temperature. The average ratio of the predicted to observed width is $0.93 \pm 0.15$ over the dataset, which has a range in width of a factor of three. Figure 1(b) also shows that most of the data points with higher triangularity tend to have larger experimental and predicted widths than data with lower triangularity. This result is consistent with previous observations that increasing the plasma triangularity can lead to larger widths $[7,8]$.

\subsection{Additional Width and Gradient Scaling Studies}

Several theoretical models predict that the pedestal width is related to the ion toroidal gyro-radius $\rho_{\mathrm{i}}$ or to the ion poloidal gyroradius $\rho_{\mathrm{i} \theta}$ [9-11]. The test of the EPED1 model provided a fairly wide range of pedestal parameters and has been used to look for evidence of these scalings. Figure 2(a) is a plot of $\Delta$ against $\left(M_{\mathrm{i}} T_{\mathrm{i}}^{\mathrm{ped}}\right)^{1 / 2} / B_{\mathrm{T}}$, proportional to $\rho_{\mathrm{i}}$, where $T_{\mathrm{i}}^{\text {ped }}$ is the pedestal value of the ion temperature and $M_{\mathrm{i}}$ is the mass of the main ions. (The filled circles are from the experiment to test the EPED1 model and the open symbols will be discussed later.) There is much scatter in the data, when plotted in this way, and a toroidal gyroradius scaling is a poor description of these results. For instance, the widths for the higher toroidal field data $\left(B_{\mathrm{T}}=1.6-2.1 \mathrm{~T}\right)$ have a different trend vs $\rho_{\mathrm{i}}$ than the lower toroidal field data $\left(B_{\mathrm{T}}=0.7-1.2 \mathrm{~T}\right)$. Thus, these data do not support any simple scaling of the width with $\rho_{\mathrm{i}}$. This result is similar to previous DIII-D results [12].
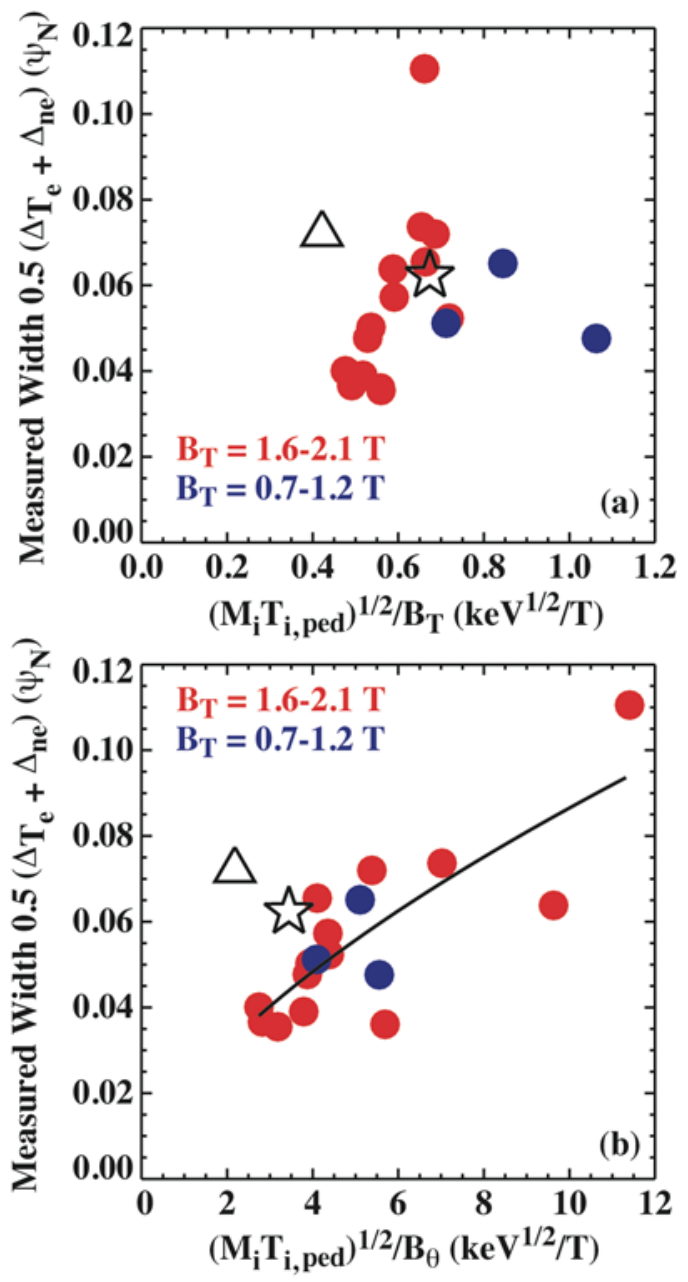

FIG. 2. Measured pedestal width vs (a) $\left(M_{\mathrm{i}} T_{\mathrm{i}}^{\mathrm{ped}}\right)^{1 / 2} / B_{\mathrm{T}}$ and (b) $\left(M_{\mathrm{i}} T_{\mathrm{i}}^{\mathrm{ped}}\right)^{1 / 2} / B_{\theta}$, proportional to ion toroidal and poloidal gyroradii, respectively. Solid curve in (b) is best fit power law to the data. Open star (diamond) is from ITER demo D (H) discharge.

Figure 2(b) is a test of a poloidal gyroradius scaling and is a plot of $\Delta$ against $\left(M_{\mathrm{i}} T_{\mathrm{i}}^{\text {ped }}\right)^{1 / 2} / B_{\theta}$, proportional to $\rho_{\mathrm{i} \theta}$, where $B_{\theta}$ is the poloidal magnetic field. This plot exhibits 
a better organization of the data than the plot versus the toroidal gyro-radius parameter. However, significant scatter remains and the plot does not provide strong evidence for either the existence or absence of a $\rho_{\mathrm{i} \theta}$ scaling. The solid line through the data is a best fit from a power law function and has an exponent of 0.6 on the abscissa. This result is very similar to a study of the large database, studied in Ref. [5], where the empirical scaling $\Delta_{\mathrm{pe}} \sim \rho_{\mathrm{i} \theta}^{0.6}$ was the best fit to the data.

Peeling-ballooning theory predicts that the maximum achievable pressure pedestal height for a fixed width has an approximately linear dependence on the product $I_{\mathrm{p}} B_{\mathrm{T}}$ [3]. This relationship implies that the maximum pressure gradient $(\nabla \mathrm{p})_{\max }$, also scales as $I_{\mathrm{p}} B_{\mathrm{T}}$, and this has been examined with the dataset used to test the

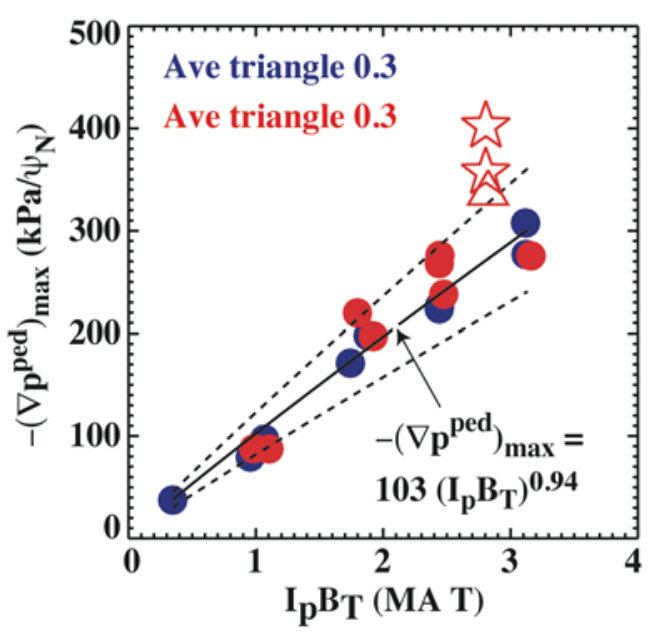

FIG. 3. Maximum total pedestal pressure gradient vs product of plasma current and toroidal field. Solid line is unity line. Dashed lines show deviations of $\pm 10 \%$ from unity. Open stars (diamond) from ITER demo $\mathrm{D}(\mathrm{H})$ discharge.

EPED1 model. Figure 3 shows the maximum measured total pressure gradient, achieved just before an ELM, plotted versus the product $I_{\mathrm{p}} B_{\mathrm{T}}$. The total pressure is determined from the measured $n_{\mathrm{e}}, T_{\mathrm{e}}$ and $T_{\mathrm{i}}$ profiles plus measurements of the carbon impurity profile, used to determine the dilution of the fuel ions. This plot organizes the data quite well. A power law fit to the data (circles only), shown in Fig. 3, provides the nearly linear scaling $(\nabla \mathrm{p})_{\max }=103$ $\left(I_{\mathrm{p}} B_{\mathrm{T}}\right)^{0.94}$, where $(\nabla \mathrm{p})_{\max }$ is measured in $\mathrm{kPa} / \psi_{\mathrm{N}}, B_{\mathrm{T}}$ is in $\mathrm{T}$ and $I_{\mathrm{p}}$ is in MA. This dataset spans a range of a factor of 3 in $B_{\mathrm{T}}, I_{\mathrm{p}}$ and in average triangularity. Figure 3 shows that for the data at $I_{\mathrm{p}} B_{\mathrm{T}} \approx 1 \mathrm{MA} \mathrm{T}$, there is no clear change in $(\nabla \mathrm{p})_{\max }$ for the low and high triangularity data. However, for higher values of $I_{\mathrm{p}} B_{\mathrm{T}}$, higher triangularity on average provides some increase in $(\nabla \mathrm{p})_{\max }$ relative to lower triangularity. This effect is most pronounced for the ITER demonstration discharges, discussed below.

\section{Pedestal Characteristics of ITER Demonstration Discharges}

In 2008, the DIII-D program performed experiments to develop and characterize discharges with the ITER baseline characteristics [13]. These discharges matched the shape, safety factor and $\beta_{\mathrm{N}}(1.8-2.0)$ of the ITER reference discharge. Good pedestal data were obtained for some of these discharges and some of these pedestal characteristics are discussed here. In particular, data from two of these "ITER demo" discharges are compared to some of the results obtained in the test of the EPED1 model. The ITER comparison data are shown as the open stars in Figs. 1(a,b), 2(a,b) and 3. In Fig. 1(a,b), the average ratio of the predictions to observations is 0.97 for pedestal height and 0.77 for the pedestal width. These results show that the model does a good job of predicting the pedestal parameters of the ITER demo discharge. Thus, the EPED1 model is expected to be a very useful tool to predict the pedestal height in ITER. From Fig. 3, the maximum pedestal pressure gradient of the ITER demo discharges is close to the fitted dependence on $I_{\mathrm{p}} B_{\mathrm{T}}$ of the other data being studied. The 
actual values of $(\nabla \mathrm{p})_{\max }$ are $\sim 10 \%-20 \%$ higher than the fit to the other data. This increased gradient might be due to the shape of the ITER demo discharges. The general conclusions from these studies are that the pedestal structure parameters of the ITER demo discharge follow the same trends as other DIII-D data and the ITER demo pedestal is quite "normal" in comparison to other ELMing H-mode discharges.

\section{Mass Scaling of Pedestal Width}

A recent experiment was performed on JT-60U to break a correlation of width measurements on both $\beta_{\theta}^{\text {ped }}$ and $\rho_{i \theta}$ by making nearly identical plasmas in $H$ and $D$ [14]. The mass difference of $\mathrm{H}$ and $\mathrm{D}$ offers a unique opportunity to break the correlation because $\beta_{\theta}^{\text {ped }}$ has no mass dependence whereas $\rho_{\mathrm{i} \theta}$ scales with ion mass $\sqrt{M_{\mathrm{i}}}$. The experiment was performed by making discharges in $\mathrm{H}$ and $\mathrm{D}$, which had the same pedestal temperatures and densities. Therefore, these discharges had the same values of $\beta_{\theta}^{\text {ped }}$ but $\rho_{\mathrm{i} \theta}$ was larger by a factor of $\sqrt{2}$ in the D plasma. There was essentially no difference observed in the ion temperature width $\Delta T_{\mathrm{i}}$ observed in the two discharges. It was concluded that the pedestal has a much weaker than linear dependence on $\rho_{i \theta}$ and that the main dependence was on $\beta_{\theta}^{\text {ped }}$.

A similar experiment was performed in DIII-D in which an $\mathrm{H}$ discharge was developed to match the deuterium ITER demo discharge run at $\beta_{\mathrm{N}}=1.8$, discussed in Sec. 3. Profiles from the best match are shown in Fig. 4. The steep gradient regions in the $n_{\mathrm{e}}, T_{\mathrm{e}}$ and total pressure profiles were nearly identical in the $\mathrm{H}$ and D plasmas. The visual impression is that the $\mathrm{H}$ pedestal was not narrower than for the D plasma and this is confirmed by measurements showing that the ratio $(\Delta)_{\mathrm{H}} /(\Delta)_{\mathrm{D}}=1.15$ where the pedestal widths $(\Delta)_{\mathrm{H}}$ and $(\Delta)_{\mathrm{D}}$ for $\mathrm{H}$ and $\mathrm{D}$, respectively, are obtained either from $0.5\left(\Delta_{\mathrm{ne}}+\Delta_{\mathrm{Te}}\right)$ or from the fits to the total pressure profile [Fig. 4(d)]. For a perfect match, a width scaling with $\left(\beta_{\theta}^{\text {ped }}\right)^{1 / 2}$ would predict that $(\Delta)_{H} /(\Delta)_{D}=1$ and a $\rho_{i \theta}$ scaling would predict that $(\Delta)_{H} /(\Delta)_{D}=0.7$. The observed ratio of 1.15 is evidence that the plasma is not following a $\rho_{\mathrm{i} \theta}$ scaling and that a scaling based on $\beta_{\theta}^{\text {ped }}$ is a better description of the data. Figure 2(a,b), plots of experimental width against parameters proportional to the ion toroidal and poloidal gyroradii $\rho_{\mathrm{i}}$ and $\rho_{\mathrm{i} \theta}$, illustrate these conclusions.
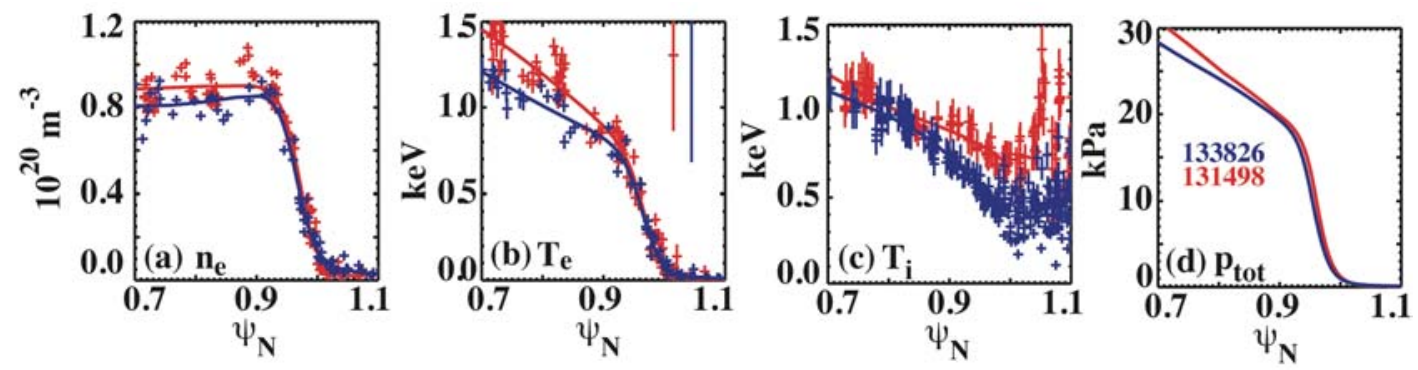

FIG 4. Comparison of profiles of (a) electron density, (b) electron temperature, (c) ion temperature (d) and total pressure from D (red) and H (blue) discharges in ITER demo configuration.

\section{Temporal Evolution of H-mode Pedestal}

Many pedestal scaling studies, including the ones presented here, reflect conditions of the pedestal just before the onset of an ELM. Modeling studies show that the maximum achievable pedestal parameters in an ELMing discharge are strongly constrained by MHD stability 
$[2,3]$. Studies of the pedestal evolution in the absence of ELMs might provide insight into the small scale transport that controls the widths and gradients of the pedestal prior to the onset of an ELM. Studies of pedestal evolution during the initial ELM-free phase, after an L-H transition, and during inter-ELM periods have been performed in beam-heated discharges in DIII-D [15] and are summarized here.

These studies show that many discharges exhibit a gradual increase of width during ELM-free and inter-ELM periods [15]. This "barrier expansion" is often most prominent in the electron density. For sufficiently long MHD-free periods, some parameters, particularly gradients, may approach a steady state. Increased heating power increases the rate at which the pedestal evolves. Often, there is a simultaneous increase of pedestal density, pedestal temperature and pedestal width. A neoclassical pedestal model predicts that the density width has the scaling $\Delta_{\mathrm{n}} \sim M_{\mathrm{i}}^{1 / 2}\left[\left(T_{\mathrm{i}}^{\mathrm{ped}}\right)^{1 / 2}-C\right] / B_{\mathrm{T}}$ where $\Delta_{\mathrm{n}}$ is measured in units of $\psi_{\mathrm{N}}$ and $C$ is a constant [16]. In some discharges, measurements of $\Delta_{\text {ne }}$ during the pedestal evolution are consistent with relationship. Initial studies also show evidence that the pedestal evolution exhibits the relationship $\Delta \sim\left(\beta_{\theta}^{\text {ped }}\right)^{1 / 2}$, as determined from empirical scaling discussed in Sec. 2. That scaling was developed to describe conditions of the pedestal at ELM onset. However, it is possible that the pedestal exhibits this scaling throughout the buildup phase to the ELM instability.

These relationships during pedestal buildup are demonstrated in Fig. 5 for the inter-ELM phase of the ITER demo discharge run at $\beta_{\mathrm{N}}=1.8$. Figure 5(a) shows that $\Delta_{\text {ne }}$ increased with $T_{\mathrm{i}}^{\text {ped }}$ during the interELM phase of this discharge. The solid line is a bestfit to the neoclassical pedestal model [16] and has the equation $\Delta_{\mathrm{ne}}=0.12\left[\left(T_{\mathrm{i}}^{\mathrm{ped}}\right)^{1 / 2}-0.42\right]$ where $\Delta_{\mathrm{ne}}$ has units of $\psi_{\mathrm{N}}$ and $T_{\mathrm{i}}^{\text {ped }}$ units of keV. Figure 5(b) shows that the pedestal also exhibited the relationship $\Delta_{\mathrm{p}} \sim$ $\left(\beta_{\theta}^{\text {ped }}\right)^{1 / 2}$ during the inter-ELM phase. The best fit of the data to $\left(\beta_{\theta}^{\text {ped }}\right)^{1 / 2}$ has the equation $\Delta=0.1\left(\beta_{\theta}^{\text {ped }}\right)^{1 / 2}$ and is shown as the solid line in Fig. 5(b). This line is a very good fit to the data and shows that pedestal width follows the $\left(\beta_{\theta}^{\text {ped }}\right)^{1 / 2}$ scaling during the pedestal buildup. The constant 0.1 is within $\sim 25 \%$ of the constant used in the width scaling for the EPED1 model.
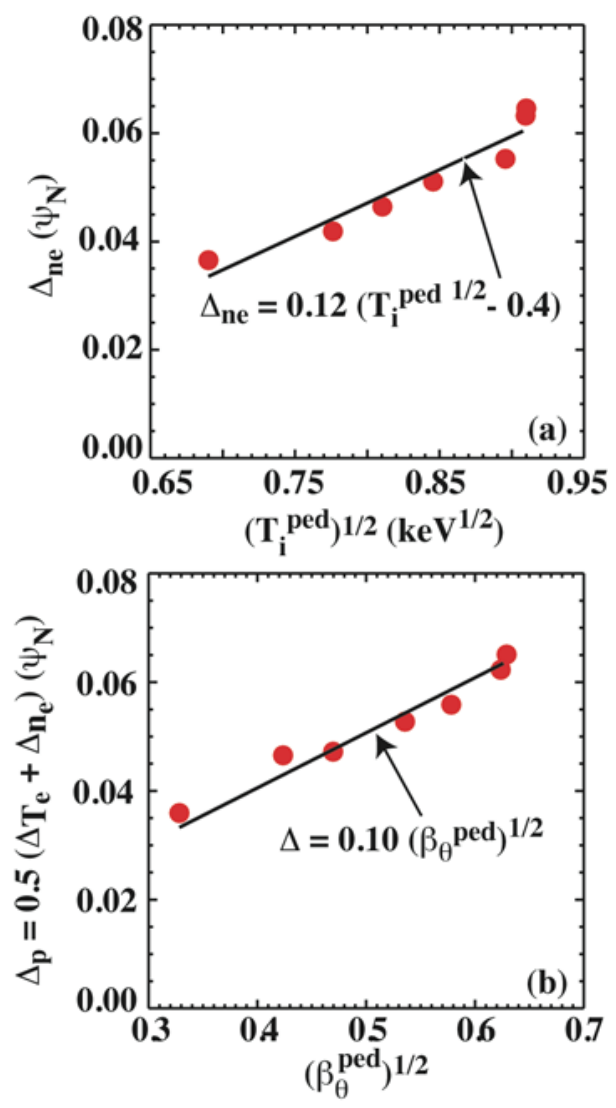

FIG. 5. (a) Electron density width vs square root of pedestal ion temperature. Line is fit to data, as described in text. (b) Pedestal width vs square of total pedestal beta poloidal. Line is fit to data, as described in text.

\section{Summary and Conclusions}

Recent DIII-D pedestal studies find that a new pedestal model, EPED1, predicts the maximum achieved pedestal width and height in Type-I ELMing discharges over a large range of DIII-D operational space, including ITER demonstration discharges. The model uses a simple 
empirical width scaling $\Delta=0.076\left(\beta_{\theta}^{\text {ped }}\right)^{1 / 2}$ which is the best existing characterization of pedestal widths in DIII-D. Width scalings based on the ion toroidal or poloidal gyroradius are much poorer descriptions of measured widths in DIII-D. These results are supported by a comparison of very similar pedestals in $\mathrm{H}$ and $\mathrm{D}$, which exhibited similar widths, a result more consistent with a width dependence on $\beta_{\theta}^{\text {ped }}$ than on a gyroradius parameter.

Expansion and growth of the H-mode barrier have been observed in the initial ELM-free H-mode and during inter-ELM periods. During this time, correlated increases have been observed in the pedestal width, pedestal pressure height and pedestal ion temperature. These observations might be evidence that the ion temperature is playing a role in setting the pedestal width during the evolution of the pedestal. However, if this is true, the physics does not appear to be as simple as a relation between the width and an ion gyroradius parameter. These data also show that the width increases with approximately the same $\left(\beta_{\theta}^{\text {ped }}\right)^{1 / 2}$ dependence as observed just before an ELM crash. If this is a general result, which remains to be demonstrated, then the empirical scaling $\Delta \sim\left(\beta_{\theta}^{\text {ped }}\right)^{1 / 2}$ discussed in Sec. 2 would be a natural consequence of the barrier expansion physics.

Due to its success in making good predictions of pedestal height for a wide range of DIII-D discharge conditions, the EPED1 model is a good candidate for making predictions of pedestal height in ITER. The scaling of pedestal width with $\left(\beta_{\theta}^{\text {ped }}\right)^{1 / 2}$ will provide more optimistic predictions for pedestal height in ITER than models based on ion gyroradii scaling. An important step for improving confidence in projections to ITER is to determine if EPED1 successfully predicts the pedestal height and width in tokamaks other than DIII-D.

\section{Acknowledgments}

This work was supported in part by the US Department of Energy under DE-FC0204ER54698, DE-AC52-07NA27344, and DE-AX05-00OR22725.

\section{References}

[1] DOYLE, E.J. et al., Nucl. Fusion 47, S18 (2007).

[2] SNYDER, P.B., et al., Fusion Energy 2008 (Proc. 22nd Int. Conf., Geneva, 2008) (Vienna: IAEA) CDROM file IT/P6-14.

[3] SNYDER, P.B., et al., Plasma Phys. Control. Fusion 46, A131 (2004).

[4] GROEBNER, R.J. et al, Nucl. Fusion 41, 1789 (2001).

[5] OSBORNE, T.H., et al, J. Nucl. Mater. 266-269, 131 (1999).

[6] OSBORNE, T.H., et al, J. Physics: Conference Series 123, 012014 (2008).

[7] LEONARD, A.W., et al., Phys. Plasmas 15, 056114 (2008).

[8] MAGGI, C.F., et al., Proc. of 35rd EPS Conf. on Plasma Physics, Hersonissos, Crete (2008).

[9] SHAING, K.C., Phys. Fluids B 4, 290 (1992).

[10] ITOH, S.-I., et al., Phys. Rev. Lett. 72, 1200 (1994).

[11] KOTSCHENREUTHER, M., et al., 1996 Proc. 16th IAEA Conf. Fusion Energy (Montreal) Vol. 2 (Vienna: IAEA) 371.

[12] FENSTERMACHER, M.E., et al., Nucl. Fusion 45, 1493 (2005).

[13] DOYLE, E.J., et al., Fusion Energy 2008 (Proc. 22nd Int. Conf. Geneva, 2008) (Vienna: IAEA) CDROM file EX/1-3.

[14] URANO H., et al., Nucl. Fusion 48, 045008 (2008).

[15] GROEBNER, R.J. et al., "Temporal Evolution of H-mode Pedestal in DIII-D," submitted to Nucl. Fusion.

[16] CHANG, C.S., et al., Bull. Am. Phys. Soc. 49, 314 (2004); CHANG, C.S., et al., Phys. Plasmas 11, 2649 (2004). 